\title{
Designing of Wireless Communication Gateway Based on Zigbee Protocol
}

\author{
Enxiu $\mathrm{CHEN}^{1,2, a,{ }^{*}, \text { Yongjun ZHANG }}{ }^{3}$, Zhang JIONG ${ }^{3}$, Wang $\mathrm{JUN}^{3}$, Changfeng \\ ZHANG ${ }^{1,2, b}$
}

${ }^{1}$ National Engineering Research Center for Agricultural Products Logistics, Jinan, 250103, China

${ }^{2}$ Shandong Key Laboratory of Storage and Transportation Preservation Technology of Agricultural Products, Jinan, 250103, China

${ }^{3}$ Shandong institute of commerce and technology, Jinan, 250103, China

aemail: project973@163.com, bemail: zcf202@163.com, ${ }^{*}$ corresponding author

Keywords: ZigBee protocol, CC2530 MCU, ZigBee/GRPS gateway software, Sim300GPRS communication module, wireless communication gateway.

Abstract. Wireless communication gateway uses CC2530 microcontroller as the core module, and utilizes Sim300 GPRS as communication module with the completion of the ZigBee/GRPS gateway hardware circuit design and application program design. The data transfer between wireless sensor network and telecommunication network by protocol conversion between different types of sensing network, and the partial management control function of the sensor network is realized. The wireless communication gateway has the versatility of the distributed target detection system, and has a good support for the development of the Internet of things, which will pave the way to mobile technique integration for application of moving calculation and management.

\section{Introduction}

ZigBee technologies is [1] [2] a kind of short distance wireless sensor network and control protocol, its working frequency is $2.4 \mathrm{GHz}$, mainly used for short distance wireless control system for the transmission of control information, the amount of data is relatively small, suitable for battery power supply system. Transmission strategy of ZigBee protocol [3] [4] has the advantage with low data exchanging cost. Therefore, this paper aims to complete the design of wireless communication gateway based on ZigBee protocol with the facility of GPRS. It can be divided into the design of hardware circuit and the design of ZigBee and GPRS gateway application [5]. The gateway design features are as following: a)The use of ZigBee network technology, wireless network can be achieved in the area of monitoring, to solve the problem of field wiring aging in local area, improve the flexibility and maintainability of the monitoring system. b) Through the GPRS network to realize the wireless connection with the remote monitoring center, to achieve data monitoring and wireless online update, can save a lot of manpower and material resources. c) The gateway has general applicability, can be used for logistics ambient monitoring, environmental monitoring, food safety monitoring, and network application of photovoltaic power station monitoring system, and has a broad application prospect in the field of monitoring. d) If the system can unify the terminal acquisition node monitoring sensor type and monitoring content in different areas, so the solution is a sound integrated technology for wireless network application.

\section{Overall System Design}

The wireless communication gateway mainly collects the data signal which is monitored by the serial port network [1], and the signal of the ZigBee module which collects the data is connected with the remote monitoring center through the wireless interface of the GPRS network module. Figure 1 shows the overall design diagram of the wireless communication gateway. The design of wireless gateway based on ZigBee protocol, need to have the relevant hardware and software, the hardware production of TI fully supports the ZigBee protocol of the CC2530 chip, the chip with the 
corresponding development kits, Z-Stack protocol stack[6] [7] and the corresponding association. The system uses CC2530 chip as the core chip, and designs a ZigBee/GPRS gateway.

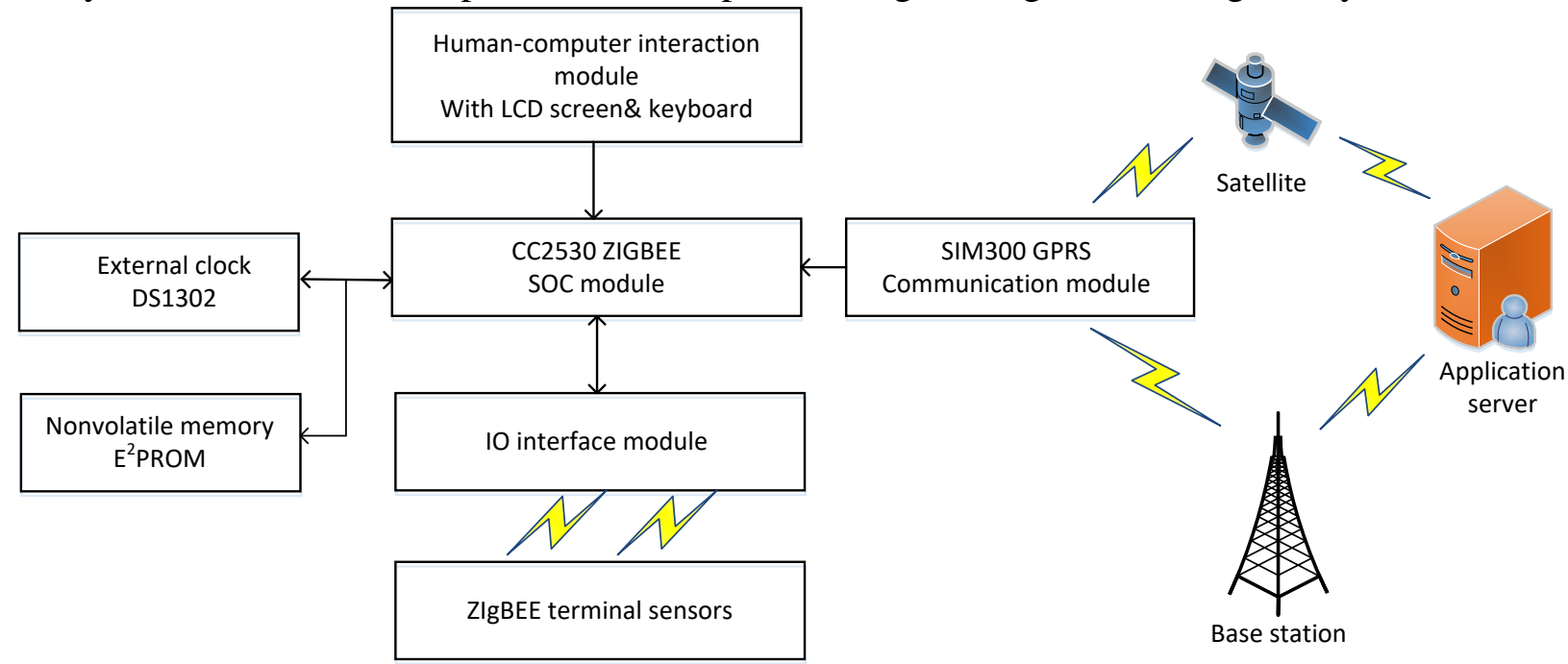

Fig. 1 The overall design block diagram of wireless communication gateway

As the gateway of wireless sensor network and the public mobile communication network[7], it has a good connection. Gateway components are as follows:

(1) CC2530 (ZigBee) provides the controller for the system (enhanced C8051) and ZigBee radio frequency module $(2.4 \mathrm{GHz})$, while controlling the conversion of other peripherals and gateway data;

(2) SIM300/GPRS communication module is connected with CC2530 through serial port, which is used for data communication between gateway and remote server;

(3) External real-time clock and E ${ }^{2}$ PROM memory are connected with CC2530 through $\mathrm{I}^{2} \mathrm{C}$ bus, and the system timing and some real time data storage are realized;

(4) The input and output communication module is connected with the terminal acquisition node through the wireless link to realize the transmission of the monitoring data and control data;

(5) The human-computer interaction module is composed of LCD and key, which is mainly used for the online inspection and maintenance of data.

\section{System Hardware Design}

CC2530 Development Board Hardware Resources Overview. CC2530 microcontroller [8] [9] is a fully compatible with the 8051 kernel, while supporting the IEEE802.15.4 protocol radio frequency MCU. With $256 \mathrm{~KB}$ of Flash, can achieve the function of ZigBee coordinator, router and child node. The core board is mainly composed of CC2530 SCM, antenna interface, crystal oscillator, ADC interface, RS interface, I/O interface, 232 corresponding liquid crystal panel etc.

(1) Power interface, to achieve the development board power supply, CC2530 microcontroller normal work requires a voltage range of 2 to $3.6 \mathrm{~V}$.

(2) Crystal oscillator circuit design, CC2530 microcontroller need two oscillator, respectively 32 $\mathrm{MHz}$ and $32.768 \mathrm{kHz}$ crystal.

(3) ADC interface, the interface can be convenient for users to AD sampling experiment.

(4) RS 232 interface, the serial communication circuit interface in the network can be connected with the Sim300/GPRS communication module.

(5) Simulation interface, the 10 pin JTAG interface can realize the program's online simulation, debugging, and Download function.

(6) Antenna interface, the antenna interface is connected to the SMA interface of the $2.4 \mathrm{GHz}$ antenna, wireless signal can be received.

(7) Extended interface, the expansion interface is the I/O port reserved by the development board, the user can use these $\mathrm{I} / \mathrm{O}$ expansion port to carry out external sensor experiments. 
(8) The corresponding liquid crystal panel, provides a 12864 dot matrix LCD module, the module with a font, easy data display.

GPRS Module Selection and Circuit Design. SIM300 module [10] is a powerful embedded TCP/IP protocol stack developed by SIMCOM Company. For SMS, voice, high-speed data transmission. The communication module is connected to the GPRS network automatically, and the communication link between the data center and the data center is established. The remote site status information of the user equipment is sent to the SCM control by sending commands.

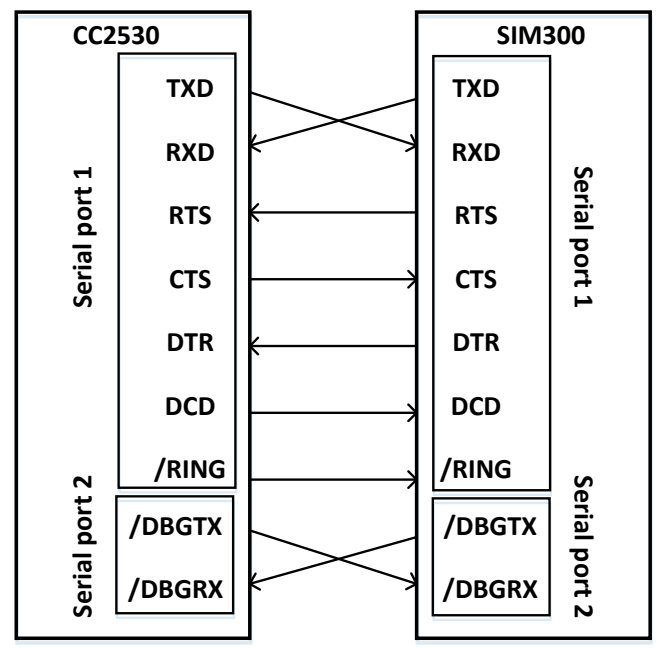

Fig. 2 SIM module and CC2530 connection

The SIM300 module and the CC2530 connection are shown in figure 2.Instructions between SIM module and CC2530 connection are as follows:

(1) The serial port 1 port has 7 lines (including the data line /TXD and /RXD, the state line /RTS and /CTS, the control line /DTR, /DCD and RING).

(2) Serial port 1 can be used as a composite signal to dial fax, GPRS service and send control module AT command. Can also use the multiplexing function, but the multiplexing function cannot use the serial port 2.

(3) 1 port, support $1200,2400,4800,9600,19200,38400,57600115200$ baud rate, the default is $115200 \mathrm{~b} / \mathrm{s}$.

(4) Auto baud support 1200, 2400, 4800, 9600, 19200, 38400, 57600115200 b/s.

(5) There are two lines on the serial port 2 (including data lines /TXD and /RXD).

(6) The serial port 2 can only be used to send AT commands, cannot be used for composite signal dialing, fax, and no multiplexing function.

(7) 2 port, support 9600, 19200, 38400, 57600115200 baud rate b/s.

In this paper, we use 163 character SPI interface LCD screen, key and LED to form the system man-machine interface, using CC2530 embedded enhanced kernel as its controller. This design uses the key and USB dual reset circuit, USB_EM_RESET which is used in the simulator control CC2530 reset 3 system software design.

\section{Architecture of Whole System}

The ZigBee/GPRS gateway software mainly realizes the establishment of ZigBee coordinator and the establishment of GPRS network, and realizes the data conversion between ZigBee and GPRS network, and the software flow chart is shown in figure 3.

First on the power system initialization, including the initialization of the ZigBee network and the GPRS module, the initialization is completed, the system into sleep until the inspection cycle to run the module processing system. Module task cycle first check whether there is a GPRS module to receive the remote monitoring center communication signal, if so, the GPRS module is the implementation of the state; if not, then the direct response. Time to determine whether the ZigBee timer. If the ZigBee query cycle timer when the node signal parameters of data acquisition and monitoring to collect each sub set, if the monitoring signal data is updated, the monitoring signal 
data sent to each node, and then determine whether there is a key signal, and update the corresponding LCD display information, storing data into a state of sleep.

ZigBee Network Programming. ZigBee protocol has been implemented in many companies have launched their own ZigBee protocol stack, which is the most prestigious Z-Stack, more widely used. Z-Stack contains almost all the functions of the network topology of the protocol stack, in the field of short distance wireless communication occupies an important position, this paper uses the Z-Stack_CC2530 version of the ZigBee protocol stack for application development.

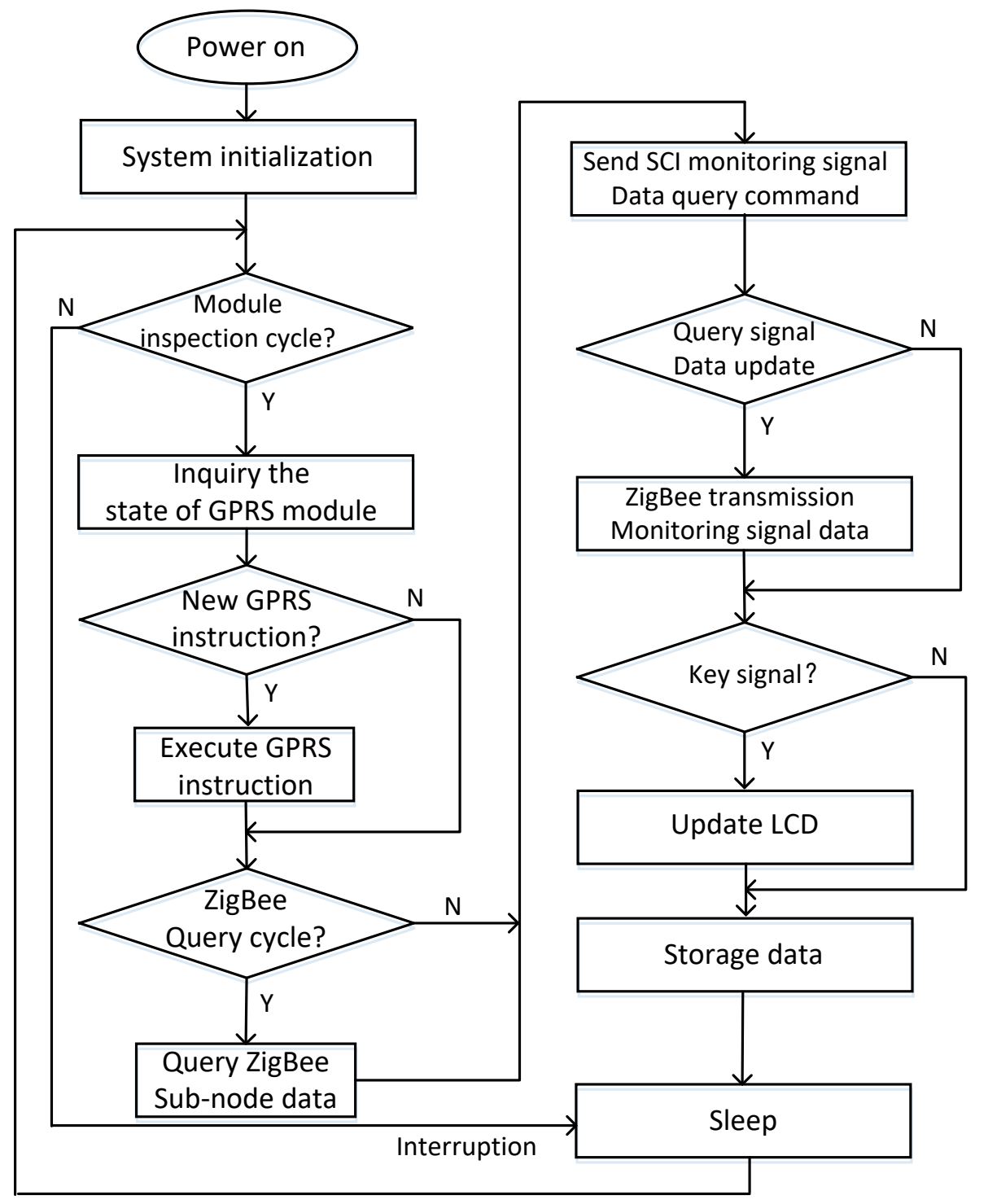

Fig.3 Workflow of embedded software

GPRS Driver Design. The workflow of the GPRS module in SIM300 is shown in figure 4. SIM300 embedded software driver includes power module, SIM300 initialization module and AT instruction function module

SIM300 Initialization. The observation on SIM300 power, network led pin on the network indicator, the network indicator light flicker frequency is $64 \mathrm{MS}$ ON/800ms OFF, working state for the SIM300 module for GPRS network, after a period of time, the flicker frequency network MS indicators for the $64 \mathrm{ON} / 3000 \mathrm{MS}$ OFF, which indicates the module is connected to the GPRS network. The length of time according to the geographical location of the signal strength, the higher the signal strength, the less time to wait, usually at $12 \mathrm{~s}$. After the SIM300 is connected to the GPRS network, the SIM300 pin of the POWERKEY receives a low pulse of greater than $1500 \mathrm{~ms}$, which is given by the microcontroller pin, as the trigger pulse of the SIM300 module.

AT Instruction. The AT instruction set is from the terminal equipment (TE) or data terminal equipment (DTE) to the terminal adapter (TA) or data terminal equipment (DCE) sent by the 
terminal adapter, TA, data terminal equipment (TE) to send AT commands to control the mobile station (MS) function, to interact with the GSM network service. Users can call, phone book, SMS, data services, text messaging, fax and other aspects of control through AT. When in use, the character is sent directly to the command, and the receiving time needs to wait and judge.

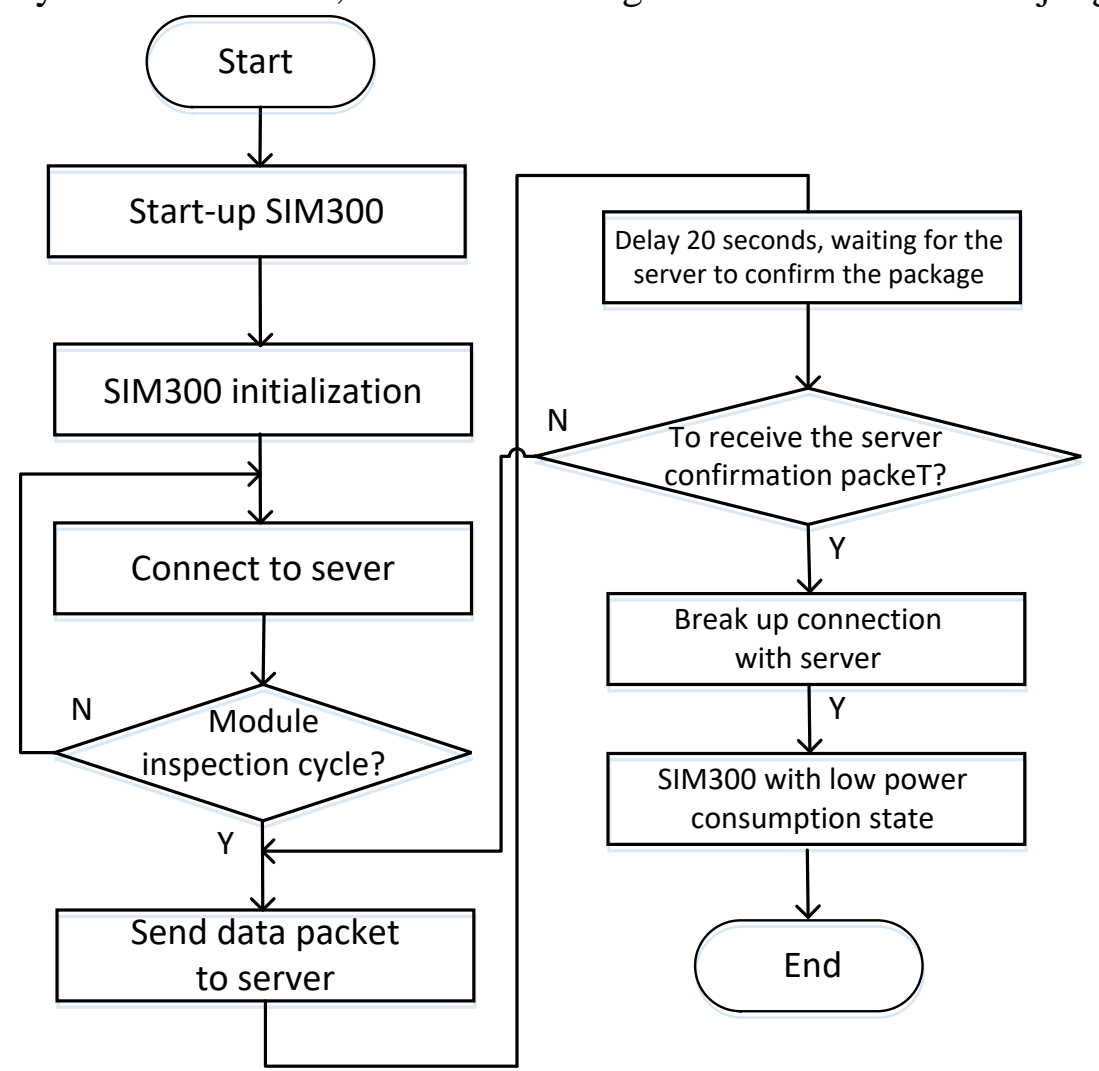

Fig.4 GPRS workflow in SIM300 module

To Establish Data Transfer to Remote Server by Using TCP. To establish a TCP connection module as the client to a remote server to initiate a TCP connection, connect successfully need client to connect to the Internet server, the server IP address is the IP address of the network (which can be obtained by dialing), runs the build server software we TCP connection. Return CONNECT OK information after successful connection. Then you can use AT+CIPSEND to send data to the server, if the server has data, the module receives data through the serial port. Use the "AT+CIPCLOSE" command to close the TCP connection.

\section{Wireless Network Remote Environment Monitoring System Based on the Gateway}

This paper uses the modular design, combined with the embedded processor and ZigBee network technology, and designed for the information system to monitor the environmental requirements for environmental applications of refrigerated compartment, the refrigerated compartment of the timing dynamic environment information acquisition and wireless transmission and remote monitoring data. In this case, the advantages of wireless communications become more apparent. Measurement of multiple parameters are often required in a certain area, for the construction of wireless sensor network communication can be achieved from the feasibility, so we combined with the characteristics of ZigBee and GPRS technology, communication gateway before using the designed wireless network monitoring system based on ZigBee/GPRS. The system has the advantages of self-organization, short distance, low power consumption, long distance transmission, and each environmental monitoring device is designed as a terminal acquisition node to form a certain distance topology network. Combined with the wireless gateway is designed for the remote transmission network equipment monitoring and terminal information collection. Through the ZigBee wireless communication gateway, the wireless communication between the coordinator node and the wireless communication module and the host computer software has been completed. 


\section{Summary}

This paper introduces a system of Embedded Gateway Based on GPRS combined with ZigBee, can realize the sensor network and the telecommunication network, Internet data forwarding, protocol conversion and management control functions, which can be widely used in the cold chain transportation, industrial control, environmental monitoring and other networking applications. The wireless sensor network to realize remote environment monitoring by network based on the modernization of processing, the move can not only meet the basic requirements of remote monitoring and measurement, but also can realize the wireless network and modern intelligent demand, has a vast application prospect.

\section{Acknowledgement}

This paper is funded by project "The state key development program of fruit and vegetable cold chain transportation logistics and quality safety control new equipment research and development" (2016YFD0400905). And It is also funded by the "The intelligent monitoring equipment of fish water-free dormancy keep live transportation” (J15LN52) from the Science and technology project of Shandong Province.

\section{References}

[1] J. van der Geer, J.A.J. Hanraads, R.A. Lupton, The art of writing a scientific article, J. Sci. Commun. 163 (2000) 51-59.

[1] N Baker ZigBee and Bluetooth strengths and weaknesses for industrial applications Computing \& Control Engineering, 2005, 16(2):20-25.

[2] A Sahafi, J Sobhi, M Sahafi, O Farhanieh, ZD Koozehkanani Ultra-low power frequency divider for 2.45 GHz ZigBee frequency synthesizer Analog Integrated Circuits and Signal Processing, 2013, 74(1):97-103.

[3] A Munir, A Gordon-Ross, S Lysecky, R Lysecky. A lightweight dynamic optimization methodology for wireless sensor networks IEEE International Conference on Wireless \& Mobile Computing, 2010, 45(2):129-136.

[4] V Potdar, A Sharif, E Chang Wireless Sensor Networks: A Survey. International Conference on Advanced Information Networking \& Applications Workshops, 2009, 38(4):636-641.

[5] SA Munir, B Ren, W Jiao, B Wang, D Xie, Mobile Wireless Sensor Network: Architecture and Enabling Technologies for Ubiquitous Computing, International Conference on Advanced Information Networking \& Applications Workshops, 2007, 2:113-120.

[6] J Xia, R Yun, K Yu, F Yin, H Wang A coordinated mechanism for multimode user equipment accessing wireless sensor network, International Journal of Grid \& Utility Computing, 2014, 5(1):1-10.

[7] Z Sun, H Cao, H Li, K Du, Y Wang, GPRS and WEB based data acquisition system for greenhouse environment,, 2006, 22(6):131-134.

[8] VA Chitre, JN Daigle, Performance of IP-based services over GPRS, Computers IEEE Transactions on, 2003, 52(6):727-741.

[9] NA Pantazis, SA Nikolidakis, DD Vergados, Energy-Efficient Routing Protocols in Wireless Sensor Networks: A Survey,Communications Surveys \& Tutorials IEEE, 2013, 15(2):551-591.

[10] JF Pliego, A Errichetti, H Wehbe-Janek, SimCom-T: An instrument to assess communication in healthcare teams, Journal of Communication in Healthcare, 2008, 1(2):168-181. 